\title{
Front Matter: Volume 10692
}

, "Front Matter: Volume 10692," Proc. SPIE 10692, Optical Fabrication, Testing, and Metrology VI, 1069201 (21 June 2018); doi: 10.1117/12.2503246

SPIE. Event: SPIE Optical Systems Design, 2018, Frankfurt, Germany 


\title{
PROCEEDINGS OF SPIE
}

\section{Optical Fabrication, Testing, and Metrology VI}

\author{
Sven Schröder \\ Roland Geyl \\ Editors
}

\section{5-17 May 2018 \\ Frankfurt, Germany}

Sponsored by

SPIE

Cosponsored by

OPTATEC (Germany)

Published by

SPIE 
The papers in this volume were part of the technical conference cited on the cover and title page. Papers were selected and subject to review by the editors and conference program committee. Some conference presentations may not be available for publication. Additional papers and presentation recordings may be available online in the SPIE Digital Library at SPIEDigitalLibrary.org.

The papers reflect the work and thoughts of the authors and are published herein as submitted. The publisher is not responsible for the validity of the information or for any outcomes resulting from reliance thereon.

Please use the following format to cite material from these proceedings:

Author(s), "Title of Paper," in Optical Fabrication, Testing, and Metrology VI, edited by Sven Schröder, Roland Geyl, Proceedings of SPIE Vol. 10692 (SPIE, Bellingham, WA, 2018) Seven-digit Article CID Number.

ISSN: 0277-786X

ISSN: 1996-756X (electronic)

ISBN: 9781510619210

ISBN: 9781510619227 (electronic)

Published by

SPIE

P.O. Box 10, Bellingham, Washington 98227-0010 USA

Telephone +1 3606763290 (Pacific Time) · Fax +1 3606471445

SPIE.org

Copyright (C) 2018, Society of Photo-Optical Instrumentation Engineers.

Copying of material in this book for internal or personal use, or for the internal or personal use of specific clients, beyond the fair use provisions granted by the U.S. Copyright Law is authorized by SPIE subject to payment of copying fees. The Transactional Reporting Service base fee for this volume is $\$ 18.00$ per article (or portion thereof), which should be paid directly to the Copyright Clearance Center (CCC), 222 Rosewood Drive, Danvers, MA 01923. Payment may also be made electronically through CCC Online at copyright.com. Other copying for republication, resale, advertising or promotion, or any form of systematic or multiple reproduction of any material in this book is prohibited except with permission in writing from the publisher. The CCC fee code is 0277$786 \mathrm{X} / 18 / \$ 18.00$.

Printed in the United States of America.

Publication of record for individual papers is online in the SPIE Digital Library.

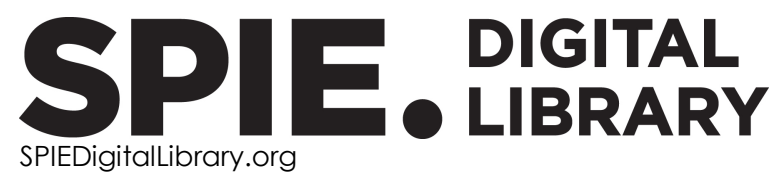

Paper Numbering: Proceedings of SPIE follow an e-First publication model. A unique citation identifier (CID) number is assigned to each article at the time of publication. Utilization of CIDs allows articles to be fully citable as soon as they are published online, and connects the same identifier to all online and print versions of the publication. SPIE uses a seven-digit CID article numbering system structured as follows:

- The first five digits correspond to the SPIE volume number.

- The last two digits indicate publication order within the volume using a Base 36 numbering system employing both numerals and letters. These two-number sets start with 00, 01, 02, 03, 04, 05, 06, 07, 08, 09, OA, OB ... 0Z, followed by 10-1Z, 20-2Z, etc. The CID Number appears on each page of the manuscript. 


\title{
Contents
}

\author{
vii Authors \\ ix Conference Committee
}

\section{OPTICAL SYSTEMS}

1069202 Optical window detachment using a 3-axis hotwire system (Invited Paper) [10692-1]

1069203 Automated centration measurement and quality inspection of aspheric lenses [10692-2]

$1069204 \quad$ Mechanical strength of optical glasses [10692-3]

\section{MICROOPTICS}

1069206 Evaluation of lenslet fabrication technologies for micro-optical array projectors [10692-5]

1069207 Improved calibration of vertical scanning optical profilometers for spherical profiles measurements [10692-6]

1069208 Holographic prism based on photo-thermo-refractive glass: new facilities for metrological application [10692-7]

\section{SPACE OPTICS}

1069209 World Space Observatory - Ultraviolet Mission: instrumentation update and status 2018 (Invited Paper) [10692-8]

10692 OA Freeform optics design and manufacturing for MicroCarb [10692-9]

10692 OB EUCLID: design, analysis, fabrication, and test of a $1.3 \mathrm{~m}$ collimator for the on-ground characterization of the EUCLID Payload Module [10692-10]

$106920 \mathrm{C}$ Additive manufacturing of metal mirrors for TMA telescope [10692-11]

10692 OD The MTG-SSA multi-purpose and multi-wavelength infrared test bench [10692-12] 


\section{GRATINGS AND FUNCTIONAL SURFACES}

10692 OE Simulation of defect-induced scattering in multilayer coatings (Invited Paper) [10692-13]

10692 OG Scattering from reflective diffraction gratings: the challenges of measurement and verification (Invited Paper) [10692-15]

$10692 \mathrm{OH} \quad$ Optical gratings with low wavefront aberrations and low straylight for enhanced spectroscopical applications [10692-16]

10692 ol Diffuse scattering of lamellar optical gratings due to line edge roughness [10692-17]

\section{FREEFORMS}

10692 OK Novel applications based on freeform technologies (Invited Paper) [10692-19]

$10692 \mathrm{OL} \quad$ Larger format freeform fabrication and metrology [10692-20]

10692 OM Improved ion beam tools for ultra-precision figure correction of curved aluminium mirror surfaces [10692-21]

10692 ON Advanced techniques for robotic polishing of aluminum mirrors [10692-22]

1069200 Shape measurement of freeform optics (Invited Paper) [10692-23]

10692 OP Advanced optical freeform substrates fabricated by ceramic 3D printing and controlled by deflectometry [10692-24]

$106920 Q \quad$ Fabrication and measurement of freeform mirror for head-up display system [10692-25]

\section{OPTICAL FABRICATION}

10692 OX Pushing SiC polishing technology for advanced applications [10692-30]

10692 OY Three wagons method to analyze ductile mode grinding processes (Invited Paper) [10692-33]

10692 OZ Ductile grinding of tungsten carbide applying standard CNC machines: a process analysis [10692-34]

$1069210 \quad$ Process control in ductile mode machining of tungsten carbide (Invited Paper) [10692-31]

$1069211 \quad$ Load controlled process window analysis of feed controlled CNC grinding [10692-32] 


\section{METROLOGY I}

1069216 Obtaining the topography of human corneas with the null-screen testing method [10692-37]

1069217 Optoelectronic system modulation transfer function measurement based on the method of summation over different frequencies harmonic functions [10692-40]

\section{METROLOGY II}

1069219 Characterization of gratings for space applications [10692-38]

\section{POSTER SESSION}

10692 1C Modeling of luminous stream in photovoltaic systems [10692-43]

10692 1D The study of sub-surface damage of fused silica after ultrasonic assisted grinding process [10692-44]

$106921 \mathrm{E}$ Analysis of method of determination of refractive index and Abbe number of lens [10692-45]

$10692 \mathrm{lF} \quad$ Effect of visibility of the fringes on the tilt measurement using a cyclic interferometer and polarization phase shifting [10692-46]

10692 1G The matters of producing optical elements for educational purposes [10692-47]

$106921 \mathrm{H} \quad$ Hyperspectrometer based on curved prism fabrication for space application [10692-48]

$106921 \mathrm{~J}$ Dynamic null-screens: a proposal for characterizing the PTSC with adaptive patterns [10692-50]

$106921 \mathrm{~K}$ Analysis of industrial production environments and derivation of a novel channel model towards optical wireless communication [10692-51] 
Proc. of SPIE Vol. 10692 1069201-6

Downloaded From: https://www.spiedigitallibrary.org/conference-proceedings-of-spie on 26 Apr 2023 Terms of Use: https://www.spiedigitallibrary.org/terms-of-use 


\section{Authors}

Numbers in the index correspond to the last two digits of the seven-digit citation identifier (CID) article numbering system used in Proceedings of SPIE. The first five digits reflect the volume number. Base 36 numbering is employed for the last two digits and indicates the order of articles within the volume. Numbers start with 00, 01, 02, 03, 04, 05, 06, 07, 08, 09, 0A, 0B...0Z, followed by 10-1Z, 20-2Z, etc.

Achour, Sakina, 19

Aguirre Aguirre, Daniel, 16 Aguirre-Caro, Jesús Alberto, $1 \mathrm{~J}$ Angervaks, A. E., 08

Armengol-Cruz, Victor Emanuel, 16 Arnold, Thomas, OM Banasch, Michael, 0 l Barré, Frédéric, OD

Baver, Jens, OM Béguelin, Jeremy, 07 Beier, Matthias, OK Belyaeva, Alina, $1 G$ Bernal, A., IF Berruée, Romain, OD Beutler, Andreas, 00 Blalock, Todd, OL Brunelle, Matthew, $\mathrm{OL}$ Burkhardt, Matthias, $\mathrm{OH}$ Butova, Daria, 1G Camargo-Fierro, Christian, 16 Campos-García, Manuel, 16, $1 \mathrm{~J}$ Chang, Keng-Shou, ID

Chaussat, G., OX

Chen, Fong-Zhi, $O Q$

Cheng, Xinbin, $\mathrm{OE}$

Cheng, Yuan-Chieh, OQ

Chou, Hsiao-Yu, OQ

Cloutrier, Christophe, OD

Dambon, Olaf, OY, OZ, 10

Damm, Christoph, OC

Dannberg, Peter, 06

DeGroote Nelson, Jessica, OL

Díaz-Uribe, José Rufino, $1 \mathrm{~J}$

Diehl, Torsten, $\mathrm{OH}$

Doan, Van Bak, 08

Doetz, Marius, $\mathrm{OY}, \mathrm{OZ}, 10$

Erdmann, Lars $\mathrm{H}$., $\mathrm{OH}$

Espinoza-Nava, Ulises, $1 \mathrm{~J}$

Etcheto, Pierre, 19

Faehnle, Oliver, OY, OZ, 10, 11

Feng, Lei, $1 \mathrm{H}$

Ferralli, Ian, OL

Fischer, Stephanie, 06

Flatt, Holger, $1 \mathrm{~K}$

Flebus, Carlo, OB

Flügel-Paul, Thomas, 0

Ganesan, A. R., IF

Gatto, Alexandre, $\mathrm{OH}$

Geyl, R., OA, OX

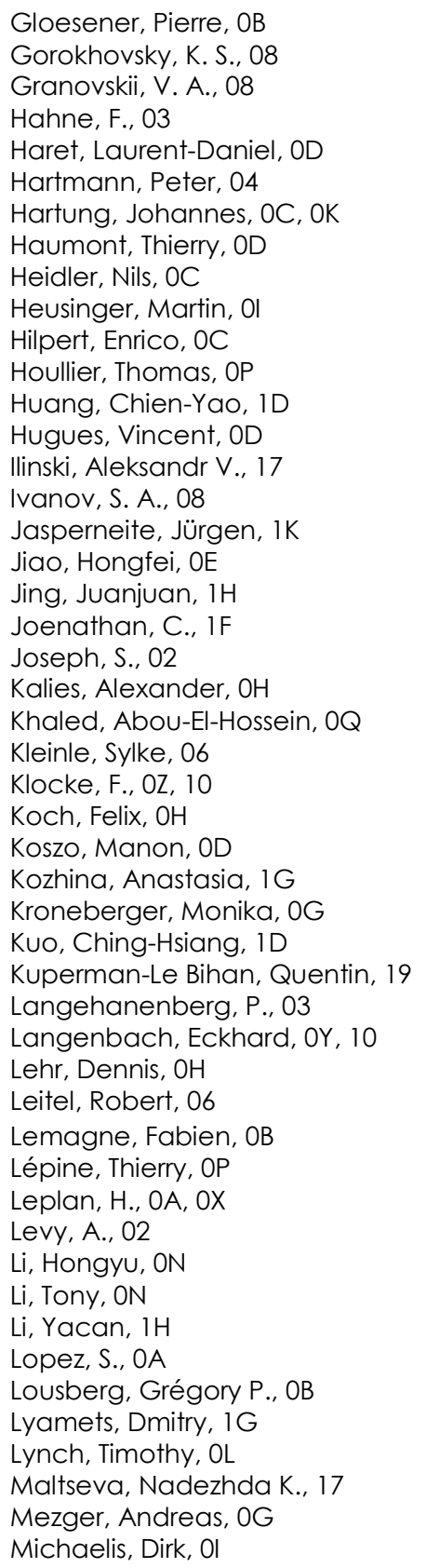


Miks, Antonin, $1 \mathrm{E}$ Moeller, Tobias, $\mathrm{OH}$ Monaci, Denis, OD Monamy, Virgile, OB Moreau, Fabien, OD Nikonorov, N. V., 08 Noell, Wilfried, 07 Novak, Jiri, 1E Novak, Pavel, 1E Nurpeisova, Diana, $1 \mathrm{G}$ Okun, R. A., 08

Orekhova, Maria K., $1 G$ Peña-Conzuelo, Andrés, $1 \mathrm{~J}$ Peng, Wei-Jei, $0 Q$ Perezyabov, Oleg A., 17 Pesch, Alexander, $\mathrm{OH}$ Peschel, Thomas, OC Pietag, Fred, OM Pirnay, Olivier, OB Plachta, Kamil, 1C Plainchamp, Bertrand, OD Pokorny, Petr, 1E Pretheesh Kumar, V. C., IF Rascher, R., OZ, 11

Reynolds, Christina, ON Rieth, Katja, OB

Riguet, Francois, OA, OD Risse, Stefan, OC, OK

Rodolfo, Jacques, OD Rousselet, Nicolas, OP Ruch, E., OA

Ryskin, A. I., 08 Sachkov, Mikhail, 09 Scharf, Toralf, 07 Schneider, Daniel, $1 \mathrm{~K}$ Schreiber, Peter, 06 Schröder, Sven, OE Shinman-Avraham, A. E., 02 Shu, Shyu-Cheng, ID Smejkal, Filip, 1E Smejkal, Michal, 1E Stübbe, Oliver, $1 \mathrm{~K}$ Surrel, Yves, OP Tolstoba, Nadezhda, IG Triebel, Peter, $\mathrm{OH}$ Trost, Marcus, OE Ulitschka, Melanie, OM Voelkel, Reinhard, 07 Vogt, C., OZ, 11 Volatier, Jean-Baptiste, OG von Lukowicz, Henrik, OC Wächter, Christoph, 06

Walker, David, ON

Wang, Zhanshan, OE

Weber, Y. B., 02

Wei, Lidong, $1 \mathrm{H}$

Yadlovker, D., 02

Yang, Lei, $1 \mathrm{H}$

Yu, Guoyu, ON

Yu, Zong-Ru, ID
Zavatskaya, Ksenia, $1 G$

Zeitner, Uwe D., Ol

Zhang, Jinlong, OE

Zhang, Lei, OE

Zhang, Wang, ON

Zheng, Xiao, ON

Zhou, Jinsong, $1 \mathrm{H}$

Zomerstein, S., 02 


\title{
Conference Committee
}

\author{
Symposium Chair
}

Wilhelm Ulrich, Carl Zeiss AG (Germany)

Symposium Co-chairs

Juan Carlos Miñano, Universidad Politécnica de Madrid (Spain)

David M. Williamson, Nikon Research Corporation of America

(United States)

Honorary Symposium Chair

Tina E. Kidger, Kidger Optics Associates (United Kingdom)

Conference Chairs

Sven Schröder, Fraunhofer-Institut für Angewandte Optik und Feinmechanik (Germany)

Roland Geyl, Safran Reosc (France)

Conference Programme Committee

Xinbin Cheng, Tongji University (China)

Sead Doric, Doric Lenses Inc. (Canada)

Angela Duparré, Fraunhofer-Institut für Angewandte Optik und

Feinmechanik (Germany)

Oliver W. Fähnle, FISBA AG (Switzerland)

Pierre Gloesener, AMOS Ltd. (Belgium)

Philippe Godefroy, Winlight System S.A. (France)

James E. Harvey, Photon Engineering LLC (United States)

François Houbre, Savimex (France)

Shay Joseph, Rafael Advanced Defense Systems Ltd. (Israel)

Sven R. Kiontke, asphericon GmbH (Germany)

François Leprêtre, Thales Angénieux S.A. (France)

Jérôme Néauport, Commissariat à l'Énergie Atomique (France)

Miloslav Ohlídal, Brno University of Technology (Czech Republic)

Manfred Prantl, Alicona Imaging GmbH (Austria)

Reinhard Völkel, SUSS MicroOptics SA (Switzerland)

Lingli Wang, Jos. Schneider Optische Werke GmbH (Germany)

Alexander Yascovich, Space Research Institute (Russian Federation) 


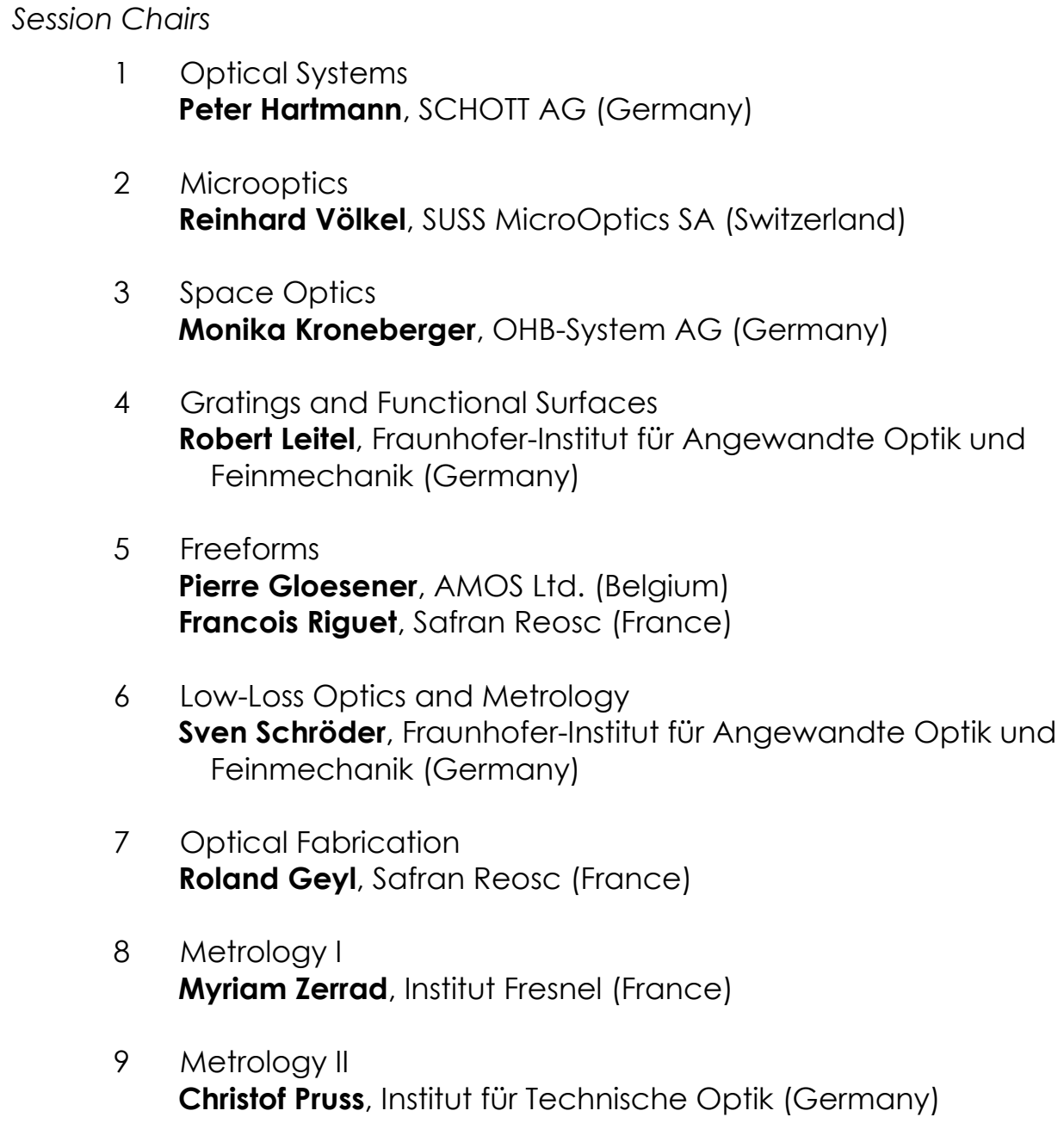

